\title{
COMPAÑIIAS DE SEGURO MARÍTIMO EN ESPAÑA (1650-1800)
}

\author{
JERÒnia PONS PONS \\ Universidad de Sevilla
}

RESUMEN: Durante el siglo XVII se produce en España una transformación del mercado asegurador asumiendo un nuevo protagonismo las compañias de seguros. Esta transformación se ha podido documentar por primera vez a partir del mercado mallorquín. A partir de la década de 1670, las compañías de seguro marítimo se convirtieron en la forma predominante de asegurar en Mallorca. Las sociedades de seguros marítimos (Caixes de Seguretat) sustituyeron a los aseguradores individuales y pronto controlaron el negocio. La comunidad conversa (chuetas) fue la responsable de su difusión. Los conversos practicaron una endogamia matrimonial, económica y social que permitió crear una comunidad cerrada. En su interior se creó una compleja red formada por compañías de seguros diferenciadas por tamaños y vinculadas por socios comunes (Caixes majors y caixons). De esta forma consiguieron una importante reducción de los costes de transacción. Este modelo continuó durante gran parte del siglo XVIII. Las características de este modelo y de estas empresas no difieren en mucho de las documentadas en otras plazas para el siglo XVIII. Estas similitudes refuerzan el modelo evolutivo por encima de la creencia de la novedad aportada con la creación de las sociedades de seguros del siglo XVIII.

PalABRAS Clave: Compañías de seguro marítimo. Redes de empresas. Conversos. Costes de transacción.

ABSTRACT: During the $17^{\text {th }}$ century an innovative leading role of insurance companies transformed the Spanish market. For the first time such renovation is documented from the Majorcan market. From the 1670's, the maritime insurance companies became the predominant method of insuring in Majorca. The maritime insurances societies (Caixes de Seguretat) replaced individuals and they soon controlled the business. The convert community (chuetas) was the driving force bebind this change. The converts practice one of marrying and carrying on economic and social activities within their community allowed to create a closed community. This «closed community» enabled them to create a complex network of insurance companies of different sizes linked through common partners (Caixes majors and caixons). In this way 
they were able to reduce the transaction costs. This model lasted during for the most part of the $18^{\text {th }}$ century. The features of the model and companies do not differ largely from those documented in other places during the $18^{\text {th }}$ century. Such similarities corroborate the evolutionary model rather than success was related with the novelty of the creation of the insurance companies in the $18^{\text {th }}$ century.

KEY WORDS: Maritime insurance companies. Network enterprise. converts. Transaction costs.

Desde su génesis en la etapa medieval hasta el siglo XIX los investigadores sobre el aspecto económico del seguro observaron la participación de amplias capas de la sociedad en el negocio asegurador. Aunque el seguro no era su única actividad sí existía cierta especialización entre determinados individuos, grupos, profesiones e incluso etnias. Todos ellos seguían la estrategia de la máxima distribución del riesgo ${ }^{1}$. Por una parte, este negocio representaba una práctica complementaria de sus actividades comerciales, financieras o artesanales principales. Por otra parte, ellos mismos establecían un límite en la cantidad asegurada en cada embarcación, por lo que era necesario recurrir a numerosos aseguradores para contratar una póliza. Los aseguradores intervenían en operaciones de seguro marítimo de forma individual o como representantes de una compañía comercial, y muy raramente como gestores de compañías de seguro especializadas. Sin embargo, la existencia de las compañías de seguro marítimas era conocida desde antiguo en la historia del seguro. Antes del siglo XVIII encontramos documentadas sociedades especializadas en operaciones de seguro en las más importantes plazas del Mediterráneo. Las más antiguas se han localizado en Génova en el siglo XV, así como en Palermo ${ }^{2}$, en Barcelona ${ }^{3}$ y Nápoles durante el siglo XVI ${ }^{4}$. Sin embargo, en estos mercados las compañías de seguros constituían una forma excepcional de operar. La forma habitual

1 Esta idea se encuentra en Boiteaux, L. A.: La Fortune de mer. Le besoin de sécurité et les débuts de l'assurance maritime, París, 1968, p. 157, en DEL TREPPO, Mario: Els mercaders catalans i l'expansió de la Corona Catalana-aragonesa al segle XV, Barcelona, 1976, pp. 373-393 y en TeNENTI, Alberto y Bratislava: Il prezzo del Rischio. L'assicurazione mediterranea vista da Ragusa (1563-1591), Roma, 1985, pp. 179-180.

2 En esta plaza GIACCHERO, Giulio: Storia delle assicurazioni marittime. L'esperienza genovese dal medievo all'età contemporanea, Genova, 1984, p. 81, cita la constitución de una compañía en 1424 , otra en 1431 y una tercera en 1433. Para el caso de Nápoles se cita una compañía fundada en esta plaza en 1569 por un periodo de un año.

3 HADZIIOSSIF, Jacqueline: «Assureurs et Assurances à Valence à l'époque des Rois Catholiques»: Horizon marins, itinéraires spirituels, vol. II, París, 1987, pp. 155-166 y GARCIA SANZ, Arcadi y Ferrer MAllol, María Teresa: Assegurances i canvis marítims medievals a Barcelona, 2 vols, Barcelona, 1983, p. 166. En este último caso se trata de una compañía constituida en 1500 por Bernat Sala, Jeroni Desplà y Joan Cortes.

4 En Nápoles Bianchini cita una compañía fundada en 1558. Nel primo centenario della Riunione Adriática di Sicurtà, 1838-1938, Trieste, 1939, p. 43. 
era la reunión coyuntural de aseguradores individuales para asegurar un barco, una mercancía o una persona. Los estudios sobre el seiscientos no modificaron la impresión de que en este siglo la organización del mercado seguía en manos de aseguradores individuales. A pesar de que se detectaron empresas de seguro en Venecia, París ${ }^{5}$ y Barcelona ${ }^{6}$, no transformaron la forma mayoritaria de operar en estas plazas. Por el contrario, en los abundantes estudios sobre la actividad comercial del siglo XVIII se detectaron numerosas compañías de seguros, algunas por acciones. Estas investigaciones contribuyeron a fijar la idea de que esta forma de asegurar era novedosa ${ }^{7}$ y afianzaron la tesis de que la especialización y la explotación del seguro de forma colectiva era un fenómeno propio de la segunda mitad del setecientos.

Sin embargo, entre la organización individual y la organización de las compañías por acciones hubo una etapa intermedia en la que las compañías de seguros colectivas tuvieron un papel fundamental. Por otra parte, si aceptamos la teoría evolutiva en la empresa ${ }^{8}$ podremos comprobar que las compañías por acciones del siglo XVIII compartirían muchas características de las empresas de seguros del XVII. Este proceso evolutivo se ha constatado hasta el momento

5 En 1680 se creó en Venecia la Compagnia d'Assicuratori in Venezia y en Francia se concedió el monopolio de los seguros a la Compagnie Générale pour les Assurances E Grosses Aventures de France en la Ville de Paris. Nel primo centenario della Riunion..., pp. 43-44.

6 Para Barcelona GiRAlt, Emili: «Família, afers i patrimoni de Jaume Cortada, mercader de Barcelona, baró de Maldà», Estudis d'Història Agrària, 6 (1986), p. 279 documenta una sociedad de seguros creada en 1636 por el corredor d'orella Jaume Cortada, Antoni Roig y el negociante Joan Tapioca. El mismo GIRALT, Emili: «La colonia mercantil francesa de Barcelona a mediados del s. XVII», Estudios de Historia Moderna, 4 (1956-59) pp. 215-278 había detectado otra compañía de seguros creada en 1645 entre Baltasar Aymerich, andreu Garoti y Pere Fises. Para la segunda mitad del seiscientos LOBATO FRANCO, Isabel: Capital Mercantil y actividad económica en la Cataluña preindustrial. Compañías y negocios en Barcelona en la segunda mitad del siglo XVII, Sevilla, 1995, p. 163 confirma la suscripción de seguros por parte de aseguradores que actúan "por compañía» sin embargo no se ha comprobado que sean compañías de seguros, sino que se trata en su mayor parte de compañías mercantiles con una gran diversidad de negocios. La autora recopila información sobre cuatro compañías de seguros entre 1689 y 1711.

A modo de Ejemplo, para las compañías de seguro en España durante el s. XVIII pueden consultarse las referencias de VILAR, Pierre: Catalunya dins l'Espanya Moderna, Barcelona, 1986, pp. 537-540, García-Baquero, Antonio: Cádiz y el Atlántico (1717-1778), Sevilla, 1976, pp. 420440, Matilla, María Jesús: «Los comienzos de la Compañía Mercantil por acciones en Barcelona (1770-80)», Primer Congrés d'Història Moderna de Catalunya, 1984, p. 737, BernAL RodríGuEZ, Antonio Miguel: La financiación de la Carrera de Indias, Sevilla, 1992, p. 502 y CARRAsCo GONZÁLEZ, Guadalupe: «El negocio de los seguros marítimos en Cádiz a finales del siglo XVIII», Hispania (Madrid), 201 (1999) pp. 269-304.

8 NelSON, Richard y WINTER, Sidney: «De una teoría evolutiva del cambio económico», en PutTerman, L. (ed.), La naturaleza económica de la empresa, Madrid, 1994 y LOPEZ García, Santiago y VALDALISO, Jesús $\mathrm{M}^{\mathrm{a}}$ (eds.), ¿Qué inventen ellos? Tecnología, empresa y cambio económico en la España contemporánea, Madrid, 1997. 
en el mercado mallorquín ${ }^{9}$ donde las fuentes han proporcionado libros contables de numerosas compañías que permiten ver su evolución y el control progresivo que ejercieron en el mercado de seguros. Su aparición y posterior dominio provocó cambios significativos en la organización del mercado. La difusión del modelo asociativo tuvo su génesis entre la comunidad de los conversos mallorquines que desarrollaron una compleja red de empresas de seguro que les permitió reducir los costes de transacción y, en definitiva, un creciente control del mercado basado en la información, la eliminación de los intermediarios, la disponibilidad de financiación y la distribución del gasto.

Este trabajo se plantea cuatro objetivos. Primero demostrar el progresivo control de mercado asegurador de compañías especializadas en este negocio en la segunda mitad del siglo XVII. Segundo, analizar sus principales características y su gestión; Tercero, estudiar la red empresarial creada por los aseguradores conversos (en Mallorca llamados chuetas) que les proporcionó el dominio del negocio hasta las persecuciones inquisitoriales iniciadas en 1678 y cuarto el análisis de la transmisión de esta organización al resto de grupos inversores en este negocio en los últimos años del seiscientos provocando la consolidación del modelo en el siglo siguiente y compararlo con el resto de plazas españolas.

\section{EL DESARRollo DE LAS SOCIEDADES DE SEGURO MARítimo EN EL MERCADO MALLORQUÍN (1650-1700).}

A partir de una amplia muestra de contratos ${ }^{10}$ de seguros localizados en los protocolos notariales se ha establecido una cronología sobre la aparición de las compañías especializadas en Mallorca. Esta periodización viene determinada en

9 Un estudio amplio sobre el desarrollo de los seguros en Mallorca, desde la evolución legislativa, el estudio del mercado, el coste del seguro, los conflictos ante el Consulado de mar y la evolución de la organización de los aseguradores en PONS PONS, Jerònia: «Cambios en la organización de los aseguradores mallorquines durante la segunda mitad del siglo XVII», Boletín de la Institución Fernán González, Año LXXIV (Burgos) Anexo al no 210 (1995) pp. 9-23, en Companyies i mercat assegurador a Mallorca (1650-1715), Palma de Mallorca, 1996 y en El sector seguros en Baleares. Empresas y empresarios en los siglos XIX y XX, Palma de Mallorca, 1998.

10 Para la confección de la muestra se han consultado los protocolos y actas de 17 notarios en el Archivo del Reino de Mallorca [=ARM]. Se han localizado 933 contratos de seguro. El total de registros notariales consultados es de 121. Los contratos de seguro se encontraban dispersos en ellos y rara vez el notario disponía de registros dedicados, única y exclusivamente, a los seguros. En la mayoría de ocasiones los libros carecían de índices temáticos. La relación de notarios con los años de los protocolos consultados es la siguiente: Antoni Amengual (1690-92), Joan Armengol (1669), Jordi Barceló (1650-1659), Llorenç Busquets (1651-679), Joan Antoni Campaner (1682-1708), Francesc Cassador (1674-1677), Francesc Femenia (1650-1653), Macià Ferrer (1650-1653), Jaume Antoni Fiol (1642-1654), Jaume Gibert (1662-1665), Antoni Joaneda (1653-1657), Antoni Moll (1662-1681), Joan Ribes (1655-1659), Joan Rotger (1675-1680), Nicolau Rubert (1664-1699), Joan Servera (1659-1690) y Gabriel Vaquer (1650-1652). 
gran parte por la actividad aseguradora de los conversos $^{11}$ ya que parte de los cambios coyunturales que sufrió el mercado asegurador mallorquín derivaron de la problemática social y económica que conoció este grupo predominante en esta actividad ${ }^{12}$.

Tras el análisis de diferentes muestras de seguro anteriores a 1650, encontramos la primera referencia a una compañía de seguro (Caixa de seguretats) en 1645, donde uno de los aseguradores afirmaba que la cantidad que cubría era per caixa. El descubrimiento de actas de constitución de caixes de seguretat en los protocolos notariales de la década siguiente junto con libros contables de estas compañías de seguros permitió conocer el funcionamiento de este tipo de compañías especializadas. La sistematización informática de mil pólizas de seguro también permitió cuantificar la participación y la evolución de estas caixes de seguretat en el mercado. A partir de este análisis cuantitativo se establecieron tres etapas en el desarrollo de las compañías de seguro en el mercado mallorquín. La primera, entre 1650 y 1677 , se centra en el crecimiento de las sociedades especializadas impulsadas por conversos. La segunda abarca entre 1678 y 1693 y viene determinada por la represión que sufren los chuetas y por la difusión del modelo colectivo entre los cristianos viejos. La tercera se establece desde 1693 en adelante en la que las sociedades aseguradoras se convirtieron en la modalidad mayoritaria, detectándose la presencia de sociedades mixtas. Las tres etapas vienen marcadas por la actividad chueta puesto que este grupo difundió, pudiendo ser el responsable de la creación, el modelo asociativo en la isla. A través de las caixes controlaron el mercado asegurador y como conse-

11 La vinculación de los judíos con el negocio del seguro se ha establecido en numerosas plazas. Entre ellas cabe citar las referencias sobre Venecia, Livorno y Ancona que cita CAIZZI, Bruno: «Assicurazione ed Economia nell'età moderna» in L'Assicurazione in Italia fino all'Unità, ANIA (Associzione Nazionale tra le imprese assicuratrici), Milano, 1975, pp. 383, 389 y 394-395.

12 Entre 1650 y 1699, a partir de la muestra de contratos, los conversos mallorquines aseguraron el $44,30 \%$ del valor asegurado y recibieron el $41,17 \%$ de las primas (PONS PONS, Jerònia, «Cambios en la organización ..., p. 14. El origen más aceptado de la palabra xueta deriva de juetó diminutivo de jueu (judío). Con este nombre se marcó en Mallorca a los descendientes de los conversos mallorquines que fueron condenados por herejes en los autos de fe de 1679 y 1691. Los miembros de este grupo se identificaron con los portadores de quince apellidos: Aguiló, Bonnín, Cortès, Forteza, Fuster, Martí, Miró, Picó, Piña, Pomar, Segura, Tarongí, Valentí, Valleriola y Valls. Para el análisis de estos acontecimientos véase BRAUSTEIN, Baruch: Els xuetes de Mallorca, Barcelona 1976 y SELKE, Angela: Vida y muerte de los chuetas en Mallorca, Madrid, 1980. Un estudio antropológico fundamental para entender la formación de este grupo y su evolución es el estudio PORQUERES GenÉ, Enric: L'endogàmia dels xuetes de Mallorca. Identitat i matrimoni en una comunitat de conversos (1435-1750), Palma, 2001. Según este autor la identificación de este grupo con los apellidos citados se realizó a partir de 1691. Sin embargo la formación del grupo es compleja. Estos apellidos no se corresponden con las de los judíos que se convirtieron al cristianismo a finales del siglo XIV y principios del siglo XV. La explicación de la concentración en estos apellidos se produjo por endogamia matrimonial. Es decir que se produjo una redefinición del grupo mediante el matrimonio. Porqueres también establece una subendogamia en el propio grupo fruto de alianzas económicas, aunque no exclusivamente. La posible preservación secreta de la ley judaica y de las costumbres judías también explicaría la formación del grupo formado por los chuetas. 
cuencia de las persecuciones inquisitoriales de 1679 y 1692 se dieron cambios significativos en el mercado. Pasemos a analizar las características y particularidades de las etapas en las que se produce la transformación del mercado.

a) El crecimiento de las sociedades especializadas impulsadas por los conversos (1650-1677). A pesar de que en los años cincuenta predominaban los aseguradores particulares durante esta década crecieron progresivamente los representantes de compañías ${ }^{13}$. Es sobre todo a partir de 1655 cuando se incrementó el número de sociedades de seguros y aumentó su proporción con respecto a los valores asegurados. En las dos décadas siguientes se desarrolló este fenómeno, apareciendo mayor número de entidades y asumiendo una mayor cuota del mercado. Entre 1650 y 1660 sólo el 25 por 100 de los aseguradores de las muestras de pólizas estudiadas operaban por compañía de seguros. Sin embargo, cubrían el 38 por 100 de las cantidades aseguradas y cobraban el 33 por 100 de las primas. En las décadas siguientes el número de aseguradores per caixa creció de forma significativa. En los años sesenta el 36,07\% de los aseguradores realizan sus operaciones en nombre de compañía, asegurando el 51,10\% del valor asegurado y cobrando el $49,62 \%$ de las primas. Ya cubrían, por tanto, la mitad del valor asegurado. Aunque en la década siguiente seguían siendo minoritarios, puesto que sólo representaban el $37,31 \%$ de los aseguradores, ya cubrían el $59,90 \%$ del valor asegurado y cobraban el 58,39\% de las primas (Cuadro $\mathrm{n}^{\circ} 1$ ).

\section{CUAdRo N ${ }^{0}$ 1. CUOTA DE MERCADO DE LA COMPAÑÍAS DE SEGURO EN EL MERCADO ASEGURADOR DE MALLORCA. 1650-1699}

\begin{tabular}{|c|c|c|c|}
\hline \multirow{2}{*}{ Año } & \multicolumn{3}{|c|}{ Porcentaje compañías de seguro } \\
\cline { 2 - 4 } & total aseguradores & Cantidad asegurada & Primas cobradas \\
\hline $1650-1660$ & 25,49 & 38,70 & 33,16 \\
\hline $1661-1670$ & 36,07 & 51,10 & 49,62 \\
\hline $1671-1680$ & 37,31 & 59,90 & 58,39 \\
\hline $1681-1690$ & 48,37 & 68,95 & 64,53 \\
\hline $1691-1699$ & 58,22 & 71,66 & 75,61 \\
\hline
\end{tabular}

Fuente: ARM, Protocolos notariales [=P.N.]. ver nota 10.

El crecimiento espectacular de las compañías de seguro en el mercado se desarrolló bajo el impulso de los conversos. Se puede establecer una clara vincula-

13 El primer suscriptor localizado que declara representar a una caixa se detecta en un seguro contratado el 11 de febrero de 1644. Se trata del converso Bartomeu Cortès. ARM, Protocolos notariales, Jaume Antoni Fiol, 5480, f. 102v. En el resto de los años cuarenta la presencia de administradores de sociedades especializadas es excepcional y siempre ligada a los conversos. 
ción entre compañías y representantes conversos ${ }^{14}$. Las Caixes de seguretats con gestores chuetas representaban el $74,53 \%$ de todos los representantes de sociedades de seguros en la década de los cincuenta, el 71,49 entre 1660 y 1669 y el 79,71\% entre 1670 y 1679 . El colectivo social de los conversos mallorquines introdujo la forma societaria en el negocio del seguro en Mallorca, aunque con el tiempo participaron otros grupos, en gran parte imitando los buenos resultados económicos de este colectivo. La media de compañías activas por año entre 1650 y 1677 era de 19 de las que 15 eran conversas y únicamente 4 estaban representadas por cristianos viejos.

En este período, por tanto, se produjo la especialización de los conversos en la suscripción de seguros participando preferentemente asociados y exclusivamente con otros conversos. La función asumida por los conversos en estas operaciones fue similar a 11 adquirida en otras actividades económicas ${ }^{15}$. El papel emergente de este colectivo en diversos planos de la economía mallorquina se interrumpió a finales de 1677 y principios del 1678. El detonante fue el encarcelamiento, secuestro de bienes y castigo de más de doscientos miembros de la comunidad conversa. Las causas que los investigadores del tema han aportado para explicar la reactivación inquisitorial son numerosas y entre ellos destaca la fuerza conseguida por este grupo en la economía de la isla ${ }^{16}$.

14 Esta clasificación se ha hecho en base al grupo social al que pertenece el tomador o representante de la compañía. Se ha aceptado que todos los tomadores chuetas representan a compañías integradas única y exclusivamente por conversos puesto que la información complementaria (actas de constitución y información contable) demuestran que esta fue la pauta antes de 1678.

15 En relación al comercio Bibiloni Amengual, Andrés: El comerç exterior de Mallorca. Homes, mercats i productes d'intercanvi (1650-1720), Palma de Mallorca, 1995, ha establecido tres etapas en la participación de los conversos. En la primera los chuetas tendrían un papel ascendente hasta 1677. Las dos décadas siguientes serían de contracción y finalmente a finales del siglo XVII y las dos primeras del XVIII recuperarían e incluso reforzarían sus presencia en el comercio exterior. En el caso concreto del comercio del lino entre 1701-1718, BibILONI AmENGUAL, Andrés: «El sector textil y el comercio exterior de Mallorca en una época de cambio (1630/1720)», Hispania (Madrid) LIX/3, núm. 203, (1999), pp. 919-920, refuerza la tesis del importante papel de este grupo en el comercio mallorquín. Su papel en el mercado del aceite en BiBILONI AMENGUAL, Andrés: Mercaders i navegants a Mallorca durant el segle XVII. L'oli com indicador del comerç mallorquí (1650-1720), Palma de Mallorca, 1992, pp. 56- 60 y como intermediarios comerciales en BIBILONI AMENGUAL, Andrés: «Intermediarios imprescindibles. Los extranjeros en la élite del comercio mallorquín del siglo XVII: el mercado del aceite», en Villar García, María Begoña y Pezzi Cristobal, Pilar (ed.), Los extranjeros en la España Moderna, tomo I, Málaga, 2003, pp. 203-215 y pp. 203-215.

16 SelKe, Angela, op. cit., pp. 126-127 argumenta una serie de factores que explican el interés represor contra este grupo: la necesidad de dinero del Tribunal de Mallorca, la presión de la Inquisición e incluso la prepotencia que demuestran los conversos respecto a los cristianos viejos. Sobre el papel de los chuetas en la economía mallorquina y el valor de las confiscaciones inquisitoriales véase MUNTANER, LLeonard.: «Los «xuetes» de Mallorca: Antisemitismo y competencia económica a finales del siglo XVII», en La Inquisición Española. Nueva visión, nuevos horizontes, Madrid, 1980 y El xuetes de Mallorca: Espai, Economia i societat a finals del segle XVII. Tesi doctoral, Barcelona, 1987. 
b) 1678-1693. Represión de los conversos y adopción del modelo colectivo por los cristianos viejos.

Los efectos del encarcelamiento y secuestro de bienes de los conversos en el campo del seguro se detectaron a partir de abril de 1678, mes en el que se anotó en las pólizas de la muestra la última intervención de las sociedades aseguradoras chuetas. Sólo se localizaron tres intervenciones a título individual en el resto del año. La retirada masiva de este grupo se manifestó en el porcentaje sobre el total asegurado ya que en este año los conversos únicamente asumieron el riesgo del $26,7 \%$. En los dos años siguientes los porcentajes disminuyeron hasta el $0,6 \%$ (1979) y 5,7\% (1680).

La desaparición del grupo dominante provocó una reestructuración en el mercado. El resultado fue sorprendente. Al estar las sociedades de seguros, casi exclusivamente, en manos de los conversos parecía que con su retirada las operaciones se redistribuirían entre los aseguradores individuales. Sin embargo no fue así. En la década de los ochenta las sociedades siguieron incrementando su papel representando el 48,37 de los aseguradores, cubriendo el 68,95\% del valor asegurado y cobrando el $64,53 \%$ de las primas.

En 1678 las sociedades formadas por cristianos viejos reactivaron sus operaciones. Las compañías de Nicolau Rubert, Jeroni Doms y Jaume Mas, operativas en 1677, asumieron el papel de máximos aseguradores que antes poseían las entidades conversas. Pero a partir de junio fueron incorporándose sociedades de nueva creación. En los tres años siguientes se produjo un boom en la fundación de entidades aseguradoras entre mercaderes, nobles, profesionales liberales, funcionarios y artesanos. Las necesidades del mercado favorecían la proliferación de sociedades aunque, la mayoría, tuvieron un corto período de supervivencia. La coyuntura favorable para la creación de nuevas sociedades especializadas perduró hasta 1683. Los acontecimientos derivados de la represión inquisitorial no detuvieron la tendencia que consolidaba la progresiva sustitución de las intervenciones particulares por operaciones de las compañías de seguro. Sin embargo, en esta década la presencia de los conversos en el mercado asegurador se redujo representando el $36,8 \%$ de todos los aseguradores y cubriendo el $33,6 \%$ de las cantidades aseguradas. El resto de grupos socioprofesionales se beneficiaron de un incremento en su cuota de mercado, sobre todo los artesanos. En la distribución socio-profesional por compañías los conversos descienden de forma importante representando el 55,34\% de todas las compañías y cubriendo el $42,38 \%$ del valor total asegurado. Entre 1678 y 1692 la media anual de sociedades aseguradoras activas era de veintiuna. De ellas doce eran conversas y el resto estaban representadas por cristianos viejos. Las distancias se habían reducido.

Los conversos se fueron reincorporando de forma lenta y progresiva a partir de 1680. En este periodo de tiempo cambiaron su estrategia de intervención en el sector. Antes de 1678 las compañías de seguros conversas eran endogámicas admitiendo sólo miembros de su comunidad. No se mezclaban con otros colec- 
tivos. Esta sectarismo permitió a la Inquisición localizar y confiscar sus bienes, incluidas las compañías aseguradoras, en el momento de la represión. Esta experiencia les indujo a primar por una parte la intervención individual y por otra la participación en sociedades de seguros junto con socios cristianos viejos ${ }^{17}$.

A partir de estos momentos las formas de constitución y gestión entre las compañías de seguros conversas y las de los cristianos viejos se fueron diluyendo, y en el mercado se hizo habitual la sociedad mixta, con participación de individuos de muy diverso oficio y condición. En este nuevo marco los conversos recuperaron parte de la cuota de mercado perdida. En el resto del período la situación de los conversos fue irregular al retomarse la presión inquisitorial entre 1689 y 1691 , que aunque cruenta, no tuvo las repercusiones económicas de la anterior.

\section{c) 1693-1700. El control del mercado por parte de las sociedades aseguradoras y la} presencia de sociedades mixtas.

El proceso de introducción de las compañías especializadas en el mercado del seguro culminó a finales de siglo cuando los representantes de las compañías representaban el 58,22\% del total de aseguradores, cubrieron el 71,66\% del valor asegurado en los contratos de la muestra y cobraron el $75,61 \%$ de las primas pagadas por los asegurados.

La formación de Caixes de seguretats se había generalizado entre todas las capas socio-profesionales. En este período los conversos recuperaron de nuevo su papel protagonista alcanzando los porcentajes más elevados de la segunda mitad del XVII (representaban el 56,4\% de todos los aseguradores y garantizaron el $54,56 \%$ de todos los valores asegurados). Existe una concentración de compañías en el sector puesto que la media se redujo a 8 compañías activas por año (cinco representadas por chuetas y tres por cristianos viejos). Sin embargo la difusión de las sociedades con una masa social mixta conduce a cuestionar la identificación del grupo socio-profesional de los tomadores con el de la masa social de la entidad.

Esta primera parte del estudio nos permite concluir que durante la segunda mitad del siglo XVII la actividad aseguradora mallorquina experimentó un cambio estructural en la organización de los aseguradores. El incremento de compañías de seguro impulsó una reducción del número de aseguradores por póliza y el incremento de las cantidades cubiertas por cada asegurador. De igual forma en este proceso de transformación se fue eliminando la figura del intermediario, en este caso el notario, obligatorio según las viejas leyes del siglo $\mathrm{XV}^{18}$, y/o del corredor. Esta desaparición proporcionó una mayor agilidad a la

17 Un ejemplo muy claro de esta nueva fórmula es la sociedad creada el 8 de diciembre de 1680 entre cinco conversos y dos destacados cargos de la Inquisición mallorquina. ARM, Protocolos notariales, Nicolau Rubert, R-165, fs. 426-432. Esta estrategia no descarta la persistencia de sociedades endogámicas.

18 Las ordenanzas mallorquinas de seguros marítimos de 1492, en su capítulo 10, declaran ilegales las pólizas que no se realicen ante notario. En la segunda mitad de siglo XVII se incrementa 
contratación de seguros. Se produjo una reducción del coste y del tiempo en la contratación. Las propias compañías tenían una mayor capacidad para reunir y cubrir cantidades cada vez más elevadas. Por tanto, podían concertar pólizas privadas dónde intervenían exclusivamente aseguradores y asegurados. Los costes de transacción se redujeron con la eliminación del coste del intermediario. Además, las propias compañías podían establecer acuerdos informales con otras para operar puesto que ya no necesitaban reunir a tantos aseguradores.

\section{CUADRO No 2. El CONTROL DE lOS CHUETAS (CONVERSOS DE MALlORCA) DEL MERCADO ASEGURADOR}

\begin{tabular}{|c|c|c|c|c|}
\hline \multirow{2}{*}{ Período } & \multicolumn{2}{|c|}{$\begin{array}{c}\text { Porcentaje } \\
\text { Sobre el total de aseguradores }\end{array}$} & \multicolumn{2}{|c|}{$\begin{array}{l}\text { Porcentaje sobre los aseguradores } \\
\text { por compañía de seguro }\end{array}$} \\
\hline & $\begin{array}{c}\text { Capital } \\
\text { asegurado }\end{array}$ & $\begin{array}{c}\text { Primas } \\
\text { cobradas }\end{array}$ & $\begin{array}{l}\text { Capital } \\
\text { asegurado }\end{array}$ & Primas cobradas \\
\hline $1650-1659$ & 46,59 & 44,47 & 74,53 & 64,86 \\
\hline $1660-1969$ & 45,58 & 45,70 & 71,49 & 65,56 \\
\hline $1970-1679$ & 36,17 & 50,22 & 79,71 & 78,25 \\
\hline $1680-1689$ & 36,79 & 33,61 & 55,34 & 42,38 \\
\hline 1690-1699 & 56,41 & 54,56 & 57,63 & 51,72 \\
\hline
\end{tabular}

Fuente: ARM, Protocolos notariales [= P.N.]. ver nota 9.

\section{LAS CAIXES DE SEGURETAT: UN MODELO DE COMPAÑÍA DE SEGUROS MARÍTI- MO EN EL SIGLO XVII.}

La proliferación de compañías de seguro en el mercado mallorquín significó un paso más en el camino hacia la profesionalización del sector. Las operaciones a partir de estos momentos estuvieron en manos de los administradores de las compañías que, aunque aún continuaban compaginando la actividad aseguradora con otros negocios o profesiones, adquirieron un contacto más directo y constante con el mercado. Los gestores eran personas más cualificadas y conocedoras del mundo mercantil.

Las actas de constitución de estas caixes de seguretat, junto con las contabilidades de varias de ellas (cuadro 3) nos permiten establecer unas características comunes: especialización (el objeto social era contratar seguros); responsabilidad colectiva, solidaria e ilimitada de su masa social; carencia de capital social inicial; indeterminación de la duración de la sociedad; fijación de un límite de

\footnotetext{
el número de pólizas privadas. Su crecimiento se refleja en el incremento de pleitos y en la documentación privada de las compañías que especifica en un importante porcentaje que la póliza es privada (PONs PONs, Jerònia: Companyies i mercat ..., pp. 33-37).
} 
cobertura por objeto asegurado en base a unos criterios establecidos por la experiencia de sus miembros; nombre religioso de la sociedad; estructura administrativa con dos cargos básicos aunque con ejemplos de mayor complejidad en la gestión y duración ilimitada de la actividad a voluntad de los socios.

En la segunda mitad del siglo XVII se adoptó este modelo de compañía de seguro cuyo objetivo era la obtener un beneficio a corto plazo. Los socios no debían aportar un capital inicial. Únicamente responsabilizarse de las derramas en caso de siniestro. Los socios cedían la gestión a los administradores. El tomador (prenedor) era el encargado de concertar y registrar los seguros. Estába obligado a respetar el límite máximo asegurable por objeto asegurado (embarcación, mercancía, persona...) establecido en los estatutos o en los acuerdos de las juntas celebradas por los $\operatorname{socios}^{19}$. Si surgía una oportunidad para contratar un seguro por valor superior el tomador debía consultar a al menos otros dos socios de la entidad. El tomador disponía de 24 horas para depositar la prima del seguro contratado en manos del cajero. En caso contrario el seguro corría por su cuenta y riesgo y no de la entidad. Debía anotar los datos de la póliza suscrita en un libro (embarcación, patrón, asegurado, destino, premio, cantidad asegurada y, en su caso, notario que registra el contrato). Por su parte, el Cajero era el responsable de la contabilidad. En un libro debía registrar, al igual que el tomador, los seguros suscritos. Además de los ingresos anotaba los pagos en concepto de siniestros, pleitos y otros gastos administrativos. Estatutariamente los gestores de las compañías de seguro recibían una compensación por su trabajo. El pago presentaba dos modalidades: en especie (una cantidad de cera que oscilaba entre 1 y dos libras) o en dinero (1 libra mallorquina).

Estos rasgos eran comunes a todas las sociedades de seguros documentadas en este período. Sin embargo los conversos crearon un modelo mucho más complejo y con importantes diferencias respecto a las sociedades de los cristianos viejos. En algunos casos la explicación de estas diferencias pasarían por factores ajenos a la estricta racionalidad económica y habría que recurrir a explicaciones antropológicas y étnico-religiosas.

Una de las prácticas de las compañías de seguro, continuada en el siglo XVIII, era la costumbre de destinar una parte de las primas para conseguir la protección religiosa. En todas las compañías de seguro del siglo XVII se desti-

19 Las actas de constitución reflejaban casi siempre el límite asegurable en cada embarcación. Esta cifra variaba en función de la capacidad financiera de los socios y su número. Por regla general las sociedades más pequeñas imponían un límite que no superaba las 100 piezas de 8 o libras mallorquinas. Las de mayor capacidad arriesgaron hasta 600 piezas de 8 o libras mallorquinas (Caixa major de 1652). Respecto a los criterios elegidos para la contratación las sociedades solían establecer una tabla de riesgos. Por ejemplo en la Caixa del Conde de Montenegro el criterio elegido era la bandera del barco. Las embarcaciones que no eran mallorquinas se aseguraban por la mitad del valor que el establecido para las isleñas. Otros criterios se establecían en función de la tipología del barco y su trayecto (Caixa de Sant Sebastià). Este aspecto se explica más extensamente en PONS PONS, Jerònia, Companyies i mercat..., pp. 214-216. 
naba un porcentaje entre un 10 y un 15 por 100 de los gastos de la sociedad a la celebración de misas para conseguir la salvaguarda del negocio, sobre todo para la protección de las embarcaciones sobre las que se había contratado el seguro $^{20}$. Sin embargo en las sociedades conversas estas cantidades se destinaban a limosnas entre los miembros pobres de su comunidad. Esta práctica era habitual en las sociedades conversas y judías europeas ${ }^{21}$. En este caso las caixes de seguretat de los criptojudíos mallorquines contribuirían a realizar la función de la cajas de comunidad judías. Esta práctica de tradición judía se camuflaría bajo las actividades de unas empresas económicas.

Por otra parte, también aparecen diferencias respecto a la inversión de los beneficios. Las compañías de seguro no establecían un capital social y por tanto los socios no debían realizar ningún desembolso inicial al constituirse la entidad. Sin embargo, en los estatutos se indicaba el procedimiento para invertir las cantidades acumuladas con el cobro de las primas de los seguros. En este caso aparecen de nuevo estrategias divergentes derivadas de prácticas socioeconómicas distintas. En los estatutos de las aseguradoras conversas se obligaba a movilizar el dinero de la caja prestando a un interés preferente a los socios de la entidad. El interés aplicado era inferior al del mercado, controlado también por el propio grupo. De esta forma los socios disponían de dinero barato para el resto de sus actividades mercantiles y/o de crédito. Sin embargo las sociedades integradas por cristianos viejos, influenciados por las costumbres de condena usuraria, optaron por la inversión en otros instrumentos mercantiles: el cambio marítimo y las inversiones en fondos de embarcaciones (parts de diners).

Las características comunes y las diferencias citadas, sin embargo, adquieren una complejidad en el caso de las entidades conversas. Entre 1650 y 1678, antes de la primera gran persecución inquisitorial, los criptojudíos impulsores de estas caixes de seguretats habían creado una red de compañías de seguro que les permitió, en tan sólo tres décadas, controlar el mercado asegurador y transformar su organización. Esta red tenía una explicación en la racionalidad económica pero seguramente no hubiera sido posible sin otros elementos propios de una comunidad cerrada ${ }^{22}$ y con una cohesión debido a elementos étnico-religiosos.

20 Véase por ejemplo como esta práctica se prolonga en las compañías de seguro catalanas que estudia VILAR, Pierre, op. cit., pp. 499-500.

21 PoRQueras GeNÉ, Enric, op. cit., p. 19 cita la persistencia de organizaciones de ayuda mutua, junto al fuerte sentimiento al grupo y la existencia de personajes considerados lideres de la comunidad como características judías que permanecen entre los chuetas. En los testamentos de los conversos es habitual la referencia a los legados piadosos de los pobres de la calle del Sagell (zona de Palma donde se concentraban parte de los conversos mallorquines). ISRAEL, Jonathan I.: La República Holandesa y el mundo bispánico, 1606-1661, Madrid, 1997, p. 236-237 explica que uno de los fines más importantes de las juntas comunales creadas por las comunidades judías en las más importantes ciudades europeas en época moderna era la creación de cajas de comunidad que procuraban ayuda básica a los judíos más pobres.

22 Granovetter, Mark: «Network Sampling: Some First Steps», The American Journal of Sociology, 81, (1976), pp. 1287-1303 ya indicada la mayor facilitar para establecer redes entre grupos pequeños y cohesionados. 


\section{LA RED DE COMPAÑÍAS DE SEGUROS CREADA POR LOS CONVERSOS}

La política matrimonial de los conversos mallorquines, basada en alianzas económicas y religiosas (práctica de costumbres semitas y observancia de leyes judaicas en la clandestinidad) había configurado un grupo bien definido y cohesionado en la Mallorca de mediados del siglo XVII. En el seno de esta comunidad se articuló una red de empresas basada en dos tipos de sociedades: Caixes majors y caixons. Ambas compartían con el resto de compañías de seguro las características comunes antes citadas. Sin embargo, los vínculos establecidos por los socios criptojudíos de ambas modalidades les confirió una nueva dimensión y les permitió en poco tiempo controlar el negocio.

Las caixes majors eran sociedades de seguros con gran número de miembros. Se dispone información de cuatro (La Caixa major de 1652, de 1659, de Sant Sebastià y de Sant Antoni) cuyos socios oscilan entre 17 y 38. Normalmente las compañías de seguros de los cristianos no superaban los diez miembros. Aunque es difícil establecer relaciones familiares entre los integrantes de las caixes majors si se ha podido confirmar que los apellidos de los socios pertenecen a las familias más ricas dentro del grupo converso: Cortès, Forteza y Martíi ${ }^{23}$. Estas caixes majors presentaban una mayor diversidad en la gestión respecto a las sociedades constituidas por los cristianos viejos: disponían de un mayor número de cargos administrativos, de la elección anual de los cargos administrativos y celebraban juntas y reuniones periódicas en las que se elegían los nuevos administradores y dónde se realizaba el balance anual.

Estas sociedades disponían de dos tomadores (prenedors) (mayor y menor), dos almoiners (mayor y menor) que se encargaban de controlar el dinero destinado a las limosnas y dos cajeros. El cajero mayor se encargaba de contabilizar el dinero que la sociedad reunía en concepto de premios y los intereses del dinero prestado. Por otra parte, el caixer de les Almoines era el responsable de contabilizar el porcentaje que se separaba de cada prima (dos sueldos por libra normalmente) que se destinaba a obras benéficas. De igual forma los conversos introdujeron en sus estatutos gestores responsables de controlar la gestión. En algunas existía el cargo de llibrer o oïdor de comptes encargado de completar un libro en el que se anotaban todos los seguros contratados y que se contrastaba, en caso de duda, con el libro del tomador. Esta figura era similar al contracompte localizada en la Caixa de cristianos viejos del tomador Nicolau Rubert.

Los estatutos de las Caixes majors establecían la celebración de juntas anuales en las que se tomaban las resoluciones de las que se levantaba acta, en las que normalmente se escogían los cargos, de entre todos los socios, cuya dura-

23 Porqueras GenÉ, Enric, op. cit., identifica en las políticas matrimoniales la unión preferente de ciertos linajes que se tienen una correspondencia con familias más ricas (Cortes, Martí, Aguiló, Forteza, Tarongí, Moya y Sureda) y matrimonios entre familias consideradas más pobres dentro del grupo (Piña, Fuster, Pomar, Picó, Bonnin y Miró) aunque siempre existieron excepciones. 
ción era anual y en la que se celebraba una fiesta. En el caso de la Caixa de Sant Sebastià, de la que disponemos información contable entre 1662 y 1678, las reuniones periódicas se celebran generalmente en enero. Estas reuniones posiblemente tuvieron una motivación más allá del estricto ámbito económico y en ellas se trataran otras cuestiones de la comunidad ${ }^{24}$. Estas caixes disponían de una gran capacidad de contratación y el limite arriesgable en cada embarcación era muy superior al resto de entidades aseguradoras de su época. Normalmente el límite se fijaba entre 200 y 300 libras o piezas de 8 , aunque el caso de la Caixa de $1652^{25}$ la cantidad llegó a ampliarse hasta las 600. El resto de entidades que operaron antes de 1678 rara vez superaron las 100 libras o piezas de 8 .

A partir de estas entidades mayores se creó una densa red aseguradora. Paralelamente a la actividad de estas caixes majors, algunos de sus socios, en pequeños grupos, constituyeron con otros individuos, perteneciente o no a otras caixes, una entidad diferente a la que denominaban caixó. Esta pequeña compañía operaba de forma independiente pero se mantenía a su vez vinculada con las Caixes majors. El calificativo mayor que acompañaba a las cajas mayores se explicaría por la subordinación de los caixons. Por ejemplo, en 1660, un miembro de la Caixa major de 1652 llamado Rafel Josep Tarongí se unió a Gabriel Piña de Gaspar y Rafel Josep Valls, socios de la Caixa de Sant Sebastià y a otros dos conversos llamados Antoni Martí y Gabriel Cortès de Francesc para constituir un caixó. De igual forma mientras eran miembros de sus respectivas Caixes Majors, Joan Antoni Cortès y Guillem Tarongí (Caixa de 1652), junto con Bartomeu Tarongí, Miquel Cortès de Josep, Rafel Forteza de Gaspar y Joan Baptista Martí (Caixa de Sant Sebastià) y Gabriel Josep Cortès (Caixa de Sant Antoni) formaron un caixó que operó entre 1665 y 1677. Se han podido documentar hasta tres caixons más entre miembros de distintas caixes majors de los que se tiene información. En el cuadro $n^{\circ} 4$ se puede ver el esquema de la red que se ha podido reconstruir a partir de la información disponible aunque posiblemente la complejidad fue mayor puesto que en los libros contables se hacen referencia a otras sociedades de las cuales no se ha podido identificar a los miembros.

Estos caixons tenían menor número de miembros, sólo dos cargos (tomador y cajero) de elección vitalicia y arriesgaban cantidades menores, nunca supera-

24 Podría corresponder a este tipo de juntas la descripción hecha por un testigo durante los procesos inquisitoriales de finales del siglo XVII. BRAUNSTEIN, Baruch, op. cit., p. 282, lo recoge: "Que en la dicha Ciudad tienen puesto destinado dentro de las calles sobredichas donde viven todos [los conversos] y en él se congregan algunas noches y hacen sus juntas a oras extraordinarias demostrando en las tales tener mucho orgullo y contento hablándose los unos a los otros desde las ventanas de sus casas y todo lo executan con mucha cautela tanto que no se fian de los de su misma Nación hasta que son ya cassados y por esto despues que los an casado les preguntan algunas personas si les an comunicado los de su Nación el secreto".

25 En los casos en que los libros contables de una compañía no identificaran su nombre o razón social y sus representantes son de elección anual se ha optado por denominarla con el nombre el primer año que tenemos noticia de su actividad. 
ban las 100 libras o piezas de 8. Podían asumir operaciones de forma independiente a las caixes majors, pero sin embargo formaban parte de su red aseguradora. La función de las caixes majors en esta red era la de concertar seguros. Una parte de la cantidad asegurada la asumía la caixa major y el resto se distribuía entre los caixons de los socios miembros de la entidad. El hecho de que los caixons estaban formados por miembros de distintas caixes majors permite establecer un sistema asegurador complejo. De esta forma, en poco tiempo, los aseguradores chuetas a través de la modalidad asociativa asumieron la parte mayor del negocio del seguro. Se especializaron. Con el tiempo una parte de los clientes dejarían de recurrir al notario y se pondrían en contacto con el tomador de una caixa major. Este a su vez, se encargaría de distribuir el seguro entre los participes de su red aseguradora. Era posible, también, que parte del seguro fuera distribuido entre los socios a título individual. En los apuntes contables de las compañías de seguro conversas se han detectado ejemplos en uno y otro sentido. Incluso se compartían cantidades entre los caixons. A modo de ejemplo se pueden citar las operaciones del caixó de 1665 representado por Miquel Cortès de Josep en cuyo libro en muchos casos se anotaba que la cantidad asegurada formaba parte de una cantidad mayor y que el resto estaba asegurado por otro caixó ${ }^{26}$.

Con este sistema se potenció el control de la actividad aseguradora entre las entidades de la comunidad conversa y, por supuesto, la eliminación del coste del intermediario, el corredor y/o notario. La incertidumbre del mercado se reducía puesto que el negocio se vuelve más directo, se comparte información y se internaliza en el sistema asegurador del grupo la decisión de la distribución del seguro que antes asumía el notario y/o el corredor. A pesar de ello, las caixes continúan participando en los seguros convencionales. Sin embargo, cada vez era más frecuente encontrar en los libros de las sociedades conversas la anotación de que de determinada póliza no se había realizado acta ante el notario. A medida que los clientes asumieron la ventaja de la contratación directa, sobre todo con las caixes conversas, y esta fue aceptada por la comunidad mercantil, las pólizas privadas adquirieron el mismo valor que las pólizas realizadas ante el notario ${ }^{27}$.

La red aseguradora conversa tenía otras funciones que contribuían a la reducción de los costes de transacción. Además de repartir las participaciones

26 ARM, Hacienda, número provisional, 258, s.f. En el seguro contratado por Esteve Conrado el 25 de febrero de 1666 se anota que las 50 piezas de 8 que aseguran son parte de 75 piezas de 8 . La 25 piezas restantes están aseguradas en el caixó nou (cajón nuevo), A partir de 1670 esta práctica se hace habitual compartiendo seguros con el caixó de Francesc Aguiló.

27 Las ordenanzas mallorquinas de 1492, en el capítulo 10, prohíben la pólizas privadas. Sin embargo, la información sobre los pleitos de seguros en el consulado de mar confirma que antes de litigar los aseguradores y asegurados declaran estar de acuerdo en utilizar la póliza privada como si fuera notarial. Esto demuestra que era una práctica aceptada. En la compañías de cristianos viejos representada por Cristòfol Seguí entre 1678 y 1681 sólo el 30 por 100 esta registrada ante notario PONS PONS, Jerònia: Companyies i mercat ..., pp. 33-37. 
entre los caixons vinculados, las caixes majors constituyeron un respaldo económico para las compañías de menor entidad, sobretodo en los momentos de falta de liquidez en el pago de un siniestro. Por último, en el caso de siniestro, los miembros de esta red afrontaron de forma conjunta el pago, tanto en los casos de acuerdo con el asegurado o cuando surgieron litigios en torno al pago de un seguro. En este último caso los pleitos se afrontaron de forma conjunta. De esta forma, las caixas y caixons conversos implicados en la pérdida de un seguro que presentaba problemas a la hora del pago se defendía conjuntamente. Tras el siniestro de una embarcación asegurada podían elevarse los gastos. Los costes de transacción en este caso se reducían si se compartían los gastos. Son numerosos los casos detectados en los cuales se puso en marcha la alianza aseguradora conversa. A modo de ejemplo citaremos el caso del siniestro de la embarcación El Beato Cayetano (El Caçador) que naufragó en 1667 en las costas de Almería cuando regresaba de Amsterdam. El seguro se pagó en 1668. Sin embargo, los aseguradores habían iniciado un litigio para la recuperación de parte de los restos. Los aseguradores, tras el pago del seguro tenían el derecho a recuperar parte de lo salvado en el naufragio. En 1670, veintidós aseguradores de la nave, algunos en nombre propio y otros en nombre de caixa, se reunieron y designaron en una acta notarial un procurador conjunto para recuperar la parte que les correspondía del salvamento ${ }^{28}$. Todos los aseguradores eran conversos, salvo uno. Con esta práctica los gastos en defensa por el impago de siniestros o recuperación de restos de un naufragio, elevados por pleitos eternos, se redujeron sustancialmente, al distribuirse entre todas las compañías de seguro afectadas que forman parte de este sistema asegurador.

\section{LA EVOLUCION DEL MODELO DE COMPAÑÍA DE SEGUROS EN EL SIGLO XVIII.}

Desconocemos que ocurrió con la red aseguradora creada por los criptojudíos tras las persecuciones de 1678 puesto que la información que disponemos de las compañías de seguro conversas proceden de los libros secuestrados por la Inquisición en este año. A partir de los datos proporcionados por las pólizas de seguro hechas ante notario conocemos el cambio de estrategia de los chuetas con la creación de sociedades mixtas. En la última década de seiscientos se puede confirmar la recuperación de las caixes de seguretat representadas por los conversos, aunque posiblemente la masa social es mixta. Es probable que tras las persecuciones inquisitoriales la reconstrucción de la red aseguradora conversa no fuera posible puesto que llevaba intrínseca connotaciones religiosas y sociales que iban más allá de los intereses económicos.

Sabemos que la modalidad colectiva se extendió entre todos los aseguradores como la forma mayoritaria de participar en el mercado de seguros. Este

\footnotetext{
28 ARM, protocolos notariales, notario Joan Servera, S-990, fs. 208v-215v.
} 
modelo perduró en el siglo XVIII. Las sociedades de seguros mallorquinas del siglo XVIII mantuvieron los mismos rasgos que sus predecesoras: a) se autodenominan caixes de seguretat; b) eran sociedades colectivas; c) disponían de los mismos gestores: tomador y cajero con las mismas responsabilidades; el tomador al igual que sus homónimas del siglo XVII disponía de un plazo de 24 horas para depositar las primas en manos del cajero; d) se descontaba un porcentaje en concepto de misas para la protección religiosa; e) no disponían de capital inicial desembolsado y f) llevaban el nombre de un santo protector.

Estas características, que las caixes de seguretat del setecientos comparten con las sociedades de seguros del siglo XVII, son similares a las descritas por diferentes autores en otras plazas españolas. Estas similitudes refuerzan la tesis evolutiva. A los elementos consolidados se le van incorporando otros novedosos propios de las compañías por acciones. En el caso mallorquín, a finales del siglo XVIII se incorporan algunos elementos propios de las compañías de seguro por acciones como la denominación de director al antiguo tomador de la sociedad, o su retribución con un porcentaje respecto a la producción de la sociedad ${ }^{29}$. De igual forma las partes en las que participan los socios se denominan acciones. Sin embargo, el paso fundamental hacia la moderna sociedad anónima no se había producido aún. No existía responsabilidad ilimitada de los socios ni se desembolsaba capital alguno. Tampoco los socios tenían responsabilidad limitada las compañías de seguros por acciones de la segunda mitad del siglo XVIII estudiadas por Matilla en Barcelona ni las investigadas por García-Baquero en Cádiz en la misma época ${ }^{30}$.

Todo ello nos induce a sostener la idea de que las compañías de seguros de la segunda mitad del siglo XVIII se denominan por acciones no eran más que una evolución del modelo analizado para el siglo XVII. Es posible que el modelo del seiscientos fuera un modelo heredado de siglos anteriores e importado por los conversos mallorquines de otras plazas por determinar. Sin embargo esta claro que la línea evolutiva no se interrumpió en el siglo XVIII. $\mathrm{Ni}$ en el caso de Mallorca ni posiblemente en otras plazas españolas en las que sea necesario un estudio más profundo del mercado asegurador del siglo XVII.

Por lo que respecta a la existencia o no de redes entre las compañías aseguradoras durante el siglo XVIII cabe indicar que con la información disponible se puede confirmar su existencia. No de una red tan compleja como la generada por los conversos pero si la existencia de una serie de compañías que posiblemente mantuvieran vínculos entre ellas en base a compartir socios comunes y los mismos gestores. Disponemos de información de al menos cinco compa-

29 Este elemento se consolida en las compañías de seguro del siglo XIX. GARCía MONTORO, Cristóbal: «Sociedades de seguros marítimos en Málaga en el siglo XIX», Homenaje al profesor Carlos Seco, Madrid 1989, p. 269 cita que en las compañías de seguros malagueñas los directores reciben como pago por su trabajo entre el 2 y el 3 por 100 de las utilidades netas de las sociedades a finales de año.

30 Matilla, María Jesús, op. cit., pp. 742-743 y García-BAQuero, Antonio, op. cit., pp. 420-425. 
ñías relacionadas entre sí (cuadro $n^{\circ}$ 5). Una parte de ellas tuvieron actividades coetáneas y fueron gestionadas por los mismos socios. La primera, Dulcíssim Cor de Jesús i Sant Ignaci, funciona desde 1767 hasta 1782. Esta formada por tres socios. El tomador de la entidad era Joseph Armati y el cajero Pau Gomila. A la muerte de este, posiblemente en 1773 , su viuda Isabel Ripoll aparecerá como cajera en una serie de nuevas caixes de seguretat creadas posteriormente: en la compañía Santo Cristo de Santa Cruz y la Purísima Concepción (1773-1782), Divino y Sagrado Corazón de Jesús y San Ignacio (1774-1779) y Sant Martí (1774?). En todas ellas el tomador es Josep Armati. Por tanto de forma coetánea los mismos gestores son responsables de cuatro caixes de seguretat, al menos, que tienen una capacidad máxima de cobertura de seguros por valor de 2600 pesos. Las composición de la masa social no es idéntica aunque una parte de los socios son comunes. En tres de ella participaba Antoni Marcel, en dos el hijo de la cajera Antoni Gomila, Domingo Pujol y Claudi Marcel. La explicación de la creación de pequeñas caixes en lugar de cajas de mayor capacidad puede explicarse en el interés por la diversificación del riesgo. El comerciante prefería diversificar sus inversiones antes que arriesgar una elevada cantidad en una única compañía de seguros puesto que cualquier siniestro elevado podía hacer fracasar la sociedad. A pesar del avance formal que supone la creación en 1787 de la compañía de seguros Santo Cristo de Santa Cruz, La Virgen su Madre Santísima de los Dolores por algunos de los socios de las entidades anteriores no se modificó esencialmente su funcionamiento. Se adoptan formas de sociedad anónima aunque los socios siguen sin tener responsabilidad limitada.

Estas caixes de seguretat funcionaban de forma independiente pero participaban de una estrategia conjunta al compartir gestores y socios: compartían información, abarataban los costes de la administración y disfrutaban de las ventajas de la especialización y del conocimiento del mercado y de los clientes por parte de los administradores. En cierta forma funcionaban de forma similar a las caixes majors conversas en el siglo XVII al distribuir las cantidades aseguradas en una póliza entre los caixons vinculados. Joseph Armati como tomador de diferentes caixes repartía la cantidad asegurada entre las diferentes entidades que gestionaba. Estas compañías carecían de la capacidad para cubrir valores elevados. Los ilustrados ${ }^{31}$ de la época recogían en sus informes las limitaciones de estas caixes de seguretats indicando que eran compañías muy débiles que no cubrían más de 4000 libras cada una. Sin embargo, este panorama no difiere en demasía de la situación descrita por Carrasco (1999), p. 295 en Cádiz donde para suscribir una póliza de 12.000 libras debían reunirse cuatro compañías. Posiblemente la formación de redes entre compañías de seguros colectivas, ya

31 Véase SARrÁ y FraU, I.: Memorias de la Real Sociedad Económica Mallorquina de Amigos de País, Primera Parte, Palma de Mallorca, 1784, pp. 44-46. En las Memorias de las Sociedad Económica de Amigos del País se describe la situación de las cajas de seguros existentes en Mallorca a finales del siglo XVIII, sus carencias y dificultades para cubrir las necesidades aseguradoras de la isla. 
sea con acuerdos informales o como el caso descrito en Mallorca compartiendo gestores comunes, permitía hacer frente a las necesidades de estos mercados. Diversificar el riesgo era por el momento la estrategia procedente de la experiencia que mejor resultado ofrecía a los aseguradores. De todas formas ya en la segunda mitad del setecientos empezaban a experimentar con la incorporación de algunos elementos de las sociedades de capital. Sin embargo, su adopción fue lenta y partió de la evolución de las sociedades ya conocidas.

\section{CONCLUSIONES}

En la segunda mitad del siglo XVII, en poco más de cuatro décadas, el mercado asegurador mallorquín se transformó. Del dominio de los aseguradores individuales se pasó a un mercado controlado por las compañías de seguro. Los principales promotores de la modalidad colectiva fueron los conversos que se organizaron en Compañías de seguros con una masa social homogénea y cerrada a los cristianos viejos. Esta organización les permitió conseguir el control del mercado. Los problemas de esta comunidad con la Inquisición y su desaparición coyuntural a finales de la década de los setenta produjo una reestructuración del mercado de la que se beneficiaron los cristianos viejos. Estos sin embargo, no incrementaron su intervención de forma individual sino que adoptan el modelo impulsado por los chuetas. Las Caixes de Seguretat se generalizan porque permitían participar en un negocio especulativo sin arriesgar grandes cantidades y sin la necesidad de conocer excesivamente el mercado. Únicamente era necesario designar unos gestores y un cajero que conocieran el negocio. Este proceso condujo a que en la década de los noventa fuera mayoritaria la modalidad colectiva y que absorbiera más del $70 \%$ de los valores asegurados. Las consecuencia de todo este cambio organizativo fue la eliminación de intermediarios entre asegurado y asegurador, el aumento de las cantidades cubiertas por asegurador y un paso más hacia la especialización.

Los protagonistas del cambio fueron los conversos mallorquines que debido a sus estrategias endogámicas y a sus prácticas religiosas secretas habían constituido una comunidad cerrada. Esta última característica les permitió crear una compleja red aseguradora cuyos pilares fueron dos tipos de sociedades de seguro (Caixes majors y caixons) vinculadas en base a socios comunes. El resultado más evidente fue la reducción de los costes de transacción. Compartieron información, financiación, gestión y gastos en los pleitos y pago de siniestros. Esta red fue posiblemente destruida tras las primeras persecuciones inquisitoriales iniciadas en 1678. Los chuetas, en un intento por la supervivencia económica se integraron en sociedades con los cristianos viejos. Los conversos mallorquines consiguieron recuperar el control del mercado de seguros a finales de siglos pero a costa de modificar su estrategia endogámica. La reconstrucción de la red aseguradora conversa no se ha podido probar. 
El modelo de caixes de seguretats persistió hasta finales del siglo XVIII. Los pasos hacia la profesionalización del negocio continuaron. En este contexto se pudo constatar la existencia de conexiones entre compañías de seguros que compartían gestores y socios como forma de reducir costes de transacción manteniendo la diversificación del riesgo tan importante para hacer frente a las incertidumbres del mercado. Por supuesto estas sociedades habían evolucionado de forma similar a otras plazas españolas. Sin embargo las guerras de finales del XVIII acabaron con muchas de estas compañías e impulsaron nuevos cambios con la generalización de las sociedades de capital. 


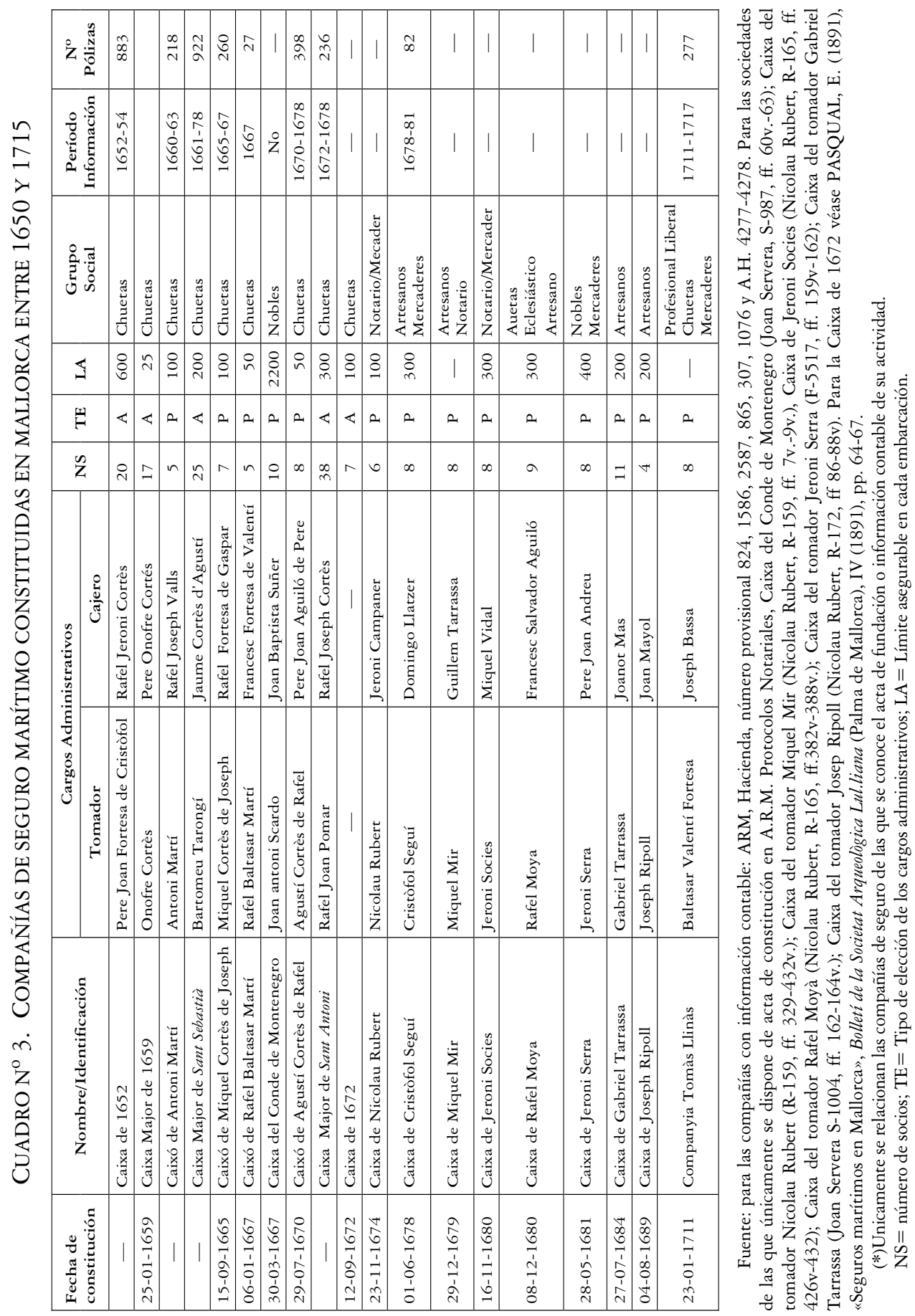

Hispania, 2007, vol. LXVII, nº. 225, enero-abril, 271-294, ISSN: 0018-2141 


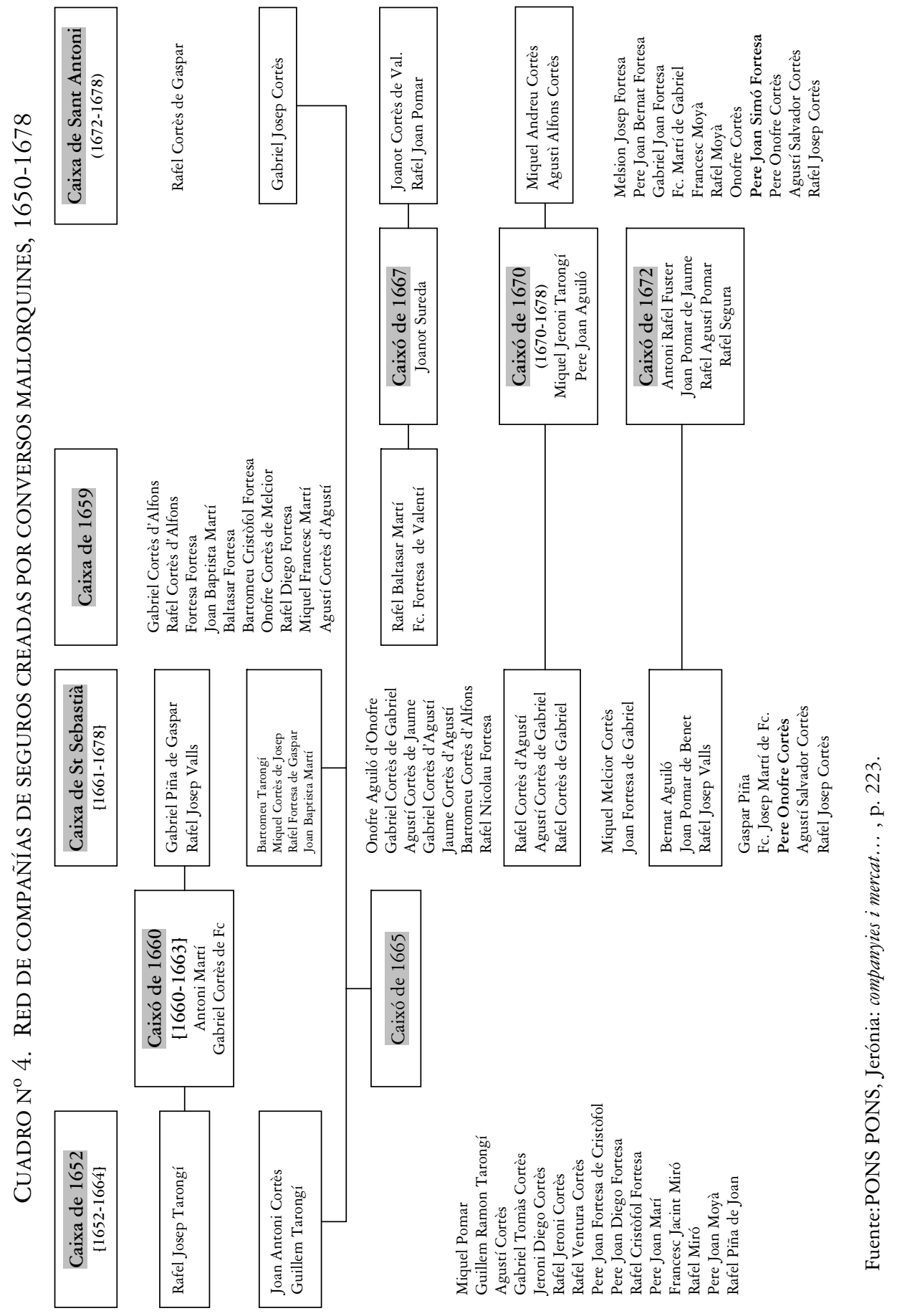




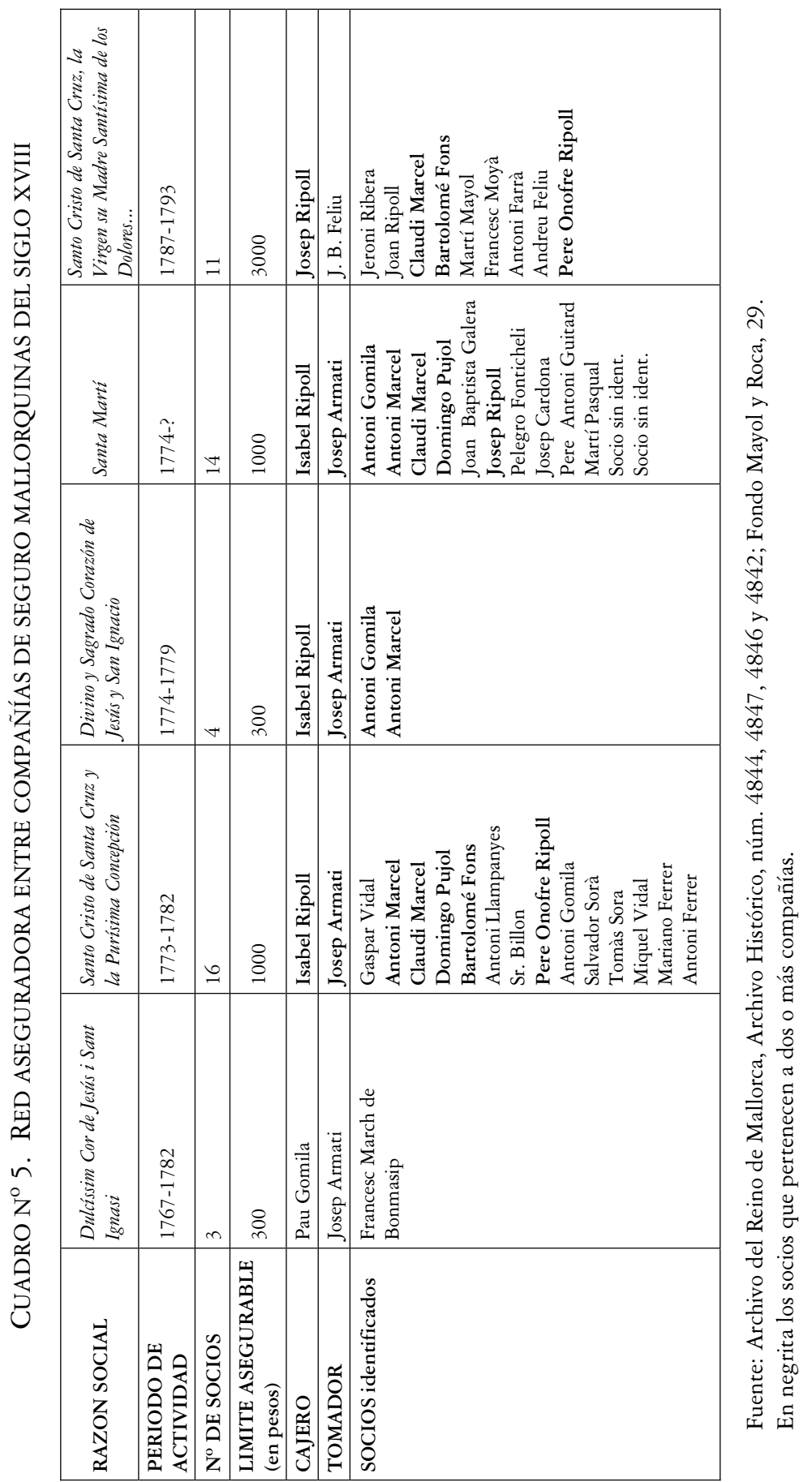

Hispania, 2007, vol. LXVII, ${ }^{\circ}$. 225, enero-abril, 271-294, ISSN: 0018-2141 